\title{
Effects of Using Fins and Carboceramics on the Solidification Characteristics of Aluminum Casting Alloys
}

\author{
Seyyed Mohammad Ali Boutorabi ${ }^{1}$, Masoud Zandira ${ }^{2}$ \\ ${ }^{1}$ School of Metallurgy and Materials Science, Center of Excellence for Advanced Materials, Iran University of Science and Tech- \\ nology, Tehran, Iran; ${ }^{2}$ Department of Materials and Manufacturing Technology, Chalmers University of Technology, Göteborg, \\ Sweden. \\ Email: boutorabi@iust.ac.ir
}

Received December $15^{\text {th }}, 2010$; revised May 21 ${ }^{\text {st }}, 2011$; accepted May $5^{\text {th }}, 2011$.

\begin{abstract}
Recent studies have shown that using the cooling fins is one of the most appropriate methods to eliminate the shrinkage porosities in the castings. In fact, Fins play the role of heat exchangers during the solidification and their usage is the same as that of chills. The cooling effect of fins is directly related to their geometry. Also the effect of fin on decreasing the time of local freezing is a function of metal's thermal conductivity, mould thermal conduction, superheating and fin dimensions. In this research, the effect of three types of fins on local solidification time of a casting of A356 and 6069 alloys as well as the effect of moisture content on cooling rate of sand moulds made of carboceramics have been studied. Results indicate that fin affects severely on solidification time and this influence is more severe in castings produced in moulding materials such as carboceramics which can absorb more moisture. The results show that carboceramics as a new refractory material decrease the solidification time significantly. This is particularly evident when the fins were used. Generally, using fins and carboceramics decrease the solidification time down to $45 \%$. This is good news for casting industry in which the mechanical properties can be easily improved using simple ways.
\end{abstract}

Keywords: Fins, Carboceramics, Solidification Time, A356, 6069

\section{Introduction}

Fin has many definitions, some of which are referred. Some references define fin as a flash attached to the casting originated as a result of cope and drag setting [1, 2]. The same definition however can be considered for flashes. In other words, there is no difference here between shrinks and fin. In the references related to thermal exchange, fin is regarded as a broad metallic sheet with high thermal conductivity and mainly as sheets with thin and long bars, acting as heat exchangers or thermal sinks $[3,4]$.

Cooling fins decrease the local solidification time and remove porosities and improve the mechanical properties $[5,6]$. Fins also increase the effective range of feeding or even may omit the need to use feeder in castings. Hence fins can be regarded as chills in casting design $[7,8]$.

Inevitable existence of flashes in moulding parts indicates the important role of fin, because flashes can be regarded as a type of fin attached to the moulding part originated circumstantially $[7,8]$. Since the first measure in fettling the castings is to remove the flashes, no study has been done on the effect of flashes as fin, considering its thermal properties.

In general, fins' properties are as follows [4,7,9-12]:

1) Fin and casting integrity in comparison with chills set up with gaps in the mould, are important from viewpoint of exact comprehensiveness towards the casting.

2) Since fin is a part of the casting, there should be no air gap in the thermal exchange route so that in addition to replicability, thermal exchange of fin, remains effective.

3) Fins do not require to be produced independently like chills.

4) Fins do not need to be separated from the returned sand for reuse. Fins are not the same as that of chills.

5) Usage of chills and their position in the mould entail limitations in some case particularly in consumable fins.

The problems of using fins can be summarized as fol- 
lows:

1) Removing the fins during fettling would increase the costs of cleaning [7,9].

2) Fin would decrease the casting efficiency. Of course it should be stated that this decline in efficiency is negligible because fins increase the solidification rate and decrease the volume of the feeder.

Studies conducted on cooling fins in moulding parts are limited. The main studies began since 1985 at Wisconsin-Madison University and continued by other researchers [13]. Most studies have been on the effects of fins in Pour-out test method in different times (Figure 1) $[3,4$, 9-13]. In this method, the melt is drained from the mould cavity after a specific time. Hence the effect of fin is studied by observing different sections of frozen samples in different times and evaluating the growth of freezing front at different points of the part, in particular near the fin. The effect of fin is indicated by Af/S in which Af is the frozen surface with fin present and $S$ is the frozen surface without the fin (Figure 2), and is indicated in a diagram based on the solidification time proportionate with $\mathrm{S} / \mathrm{T}$ in which $\mathrm{S}$ is the frozen surface without fin and $\mathrm{T}$ is the thickness of the fins (Figure 3) [1,3,9-12].

Superheating is a factor affecting the role of fin [7]. Any change in superheating will change the fin's effective range which can be seen in Figure 3 .

The effect of fin on castings made of lead and copper
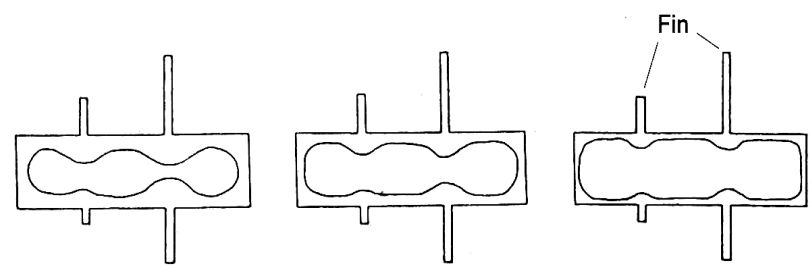

Figure 1 Schematic drawing of some of the results of Pourout test, the time of pouring the melt is increased from right to left respectively [11].

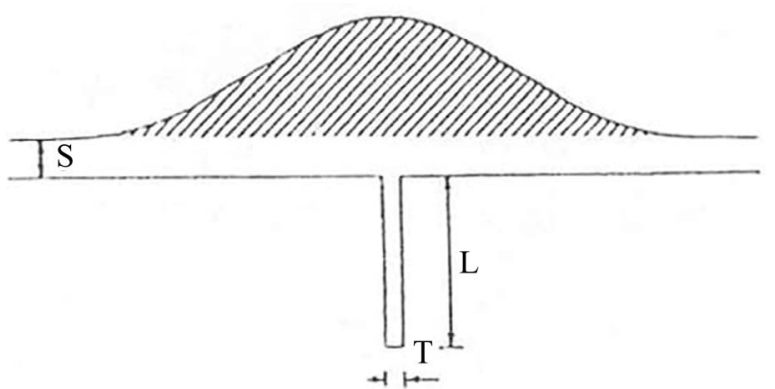

Figure 2. Schematic drawing of the cross section of solidification front of the casting with fin, $S$ is the frozen surface without fin, $T$ is the thickness of the fin, $L$ is its length $[1,4$, 11].

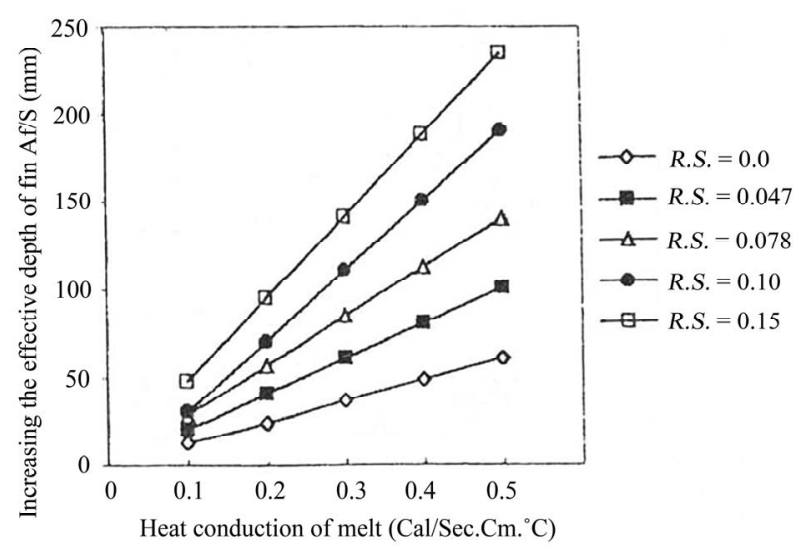

Figure 3. Diagram of considering the effect of heat conduction of melt on increasing the effective depth of fin [4], R.S $=\Delta \mathrm{T} / \mathrm{Tm},(\Delta \mathrm{T}$ is superheating and $\mathrm{Tm}$ is melting point $)$.

indicates that the ratio Af/S will be maximized as the $\mathrm{S} / \mathrm{T}$ ratio increases and then it will decrease. In other words, one can conclude that if the fin thickness is fixed, its effectiveness will not exceed than a specific range $[12,13]$. Sand and Cavity, Carboceramics

The cavity is a place where the molten metal solidifies. Mechanical properties and metallurgical structure of castings depend on factors including melt quality, casting temperature, inoculation efficiency, mould cavity characteristics, heat transfer, etc.

Heat transfer from filled mould depends on different factors the most important of which is the sand moisture. Of course type of the refractory materials, type of additives like bentonite, coal, size and shape of sand granules are also important. But sand moisture plays a dominant role in transferring the heat from mould to the environment.

In old fashioned and modern moulding systems, the moisture content ranges between 3.5 and 5 percent. Exceeding this limit would decrease the mould characteristics. Originally, in moulding, the ratio of the bentonite used to the moisture content is defined as an index which is around 2;

Clay/Water $=2$

If the bentonite increases, the sinter point of the mould will decrease. And if the moisture content decreases, the mould's strength will decrease. Also in this case, the gas porosity will increase. The general behavior of the mould characteristics due to the bentonite and moisture content ratios is shown in Figure 4.

Now if the moisture content increases, the cooling rate will increase. Recently a new type of artificial sand is presented in European market that in addition to the fact that sand granules are nearly round, there are porosities on them. It seems that considering the specific coordi- 


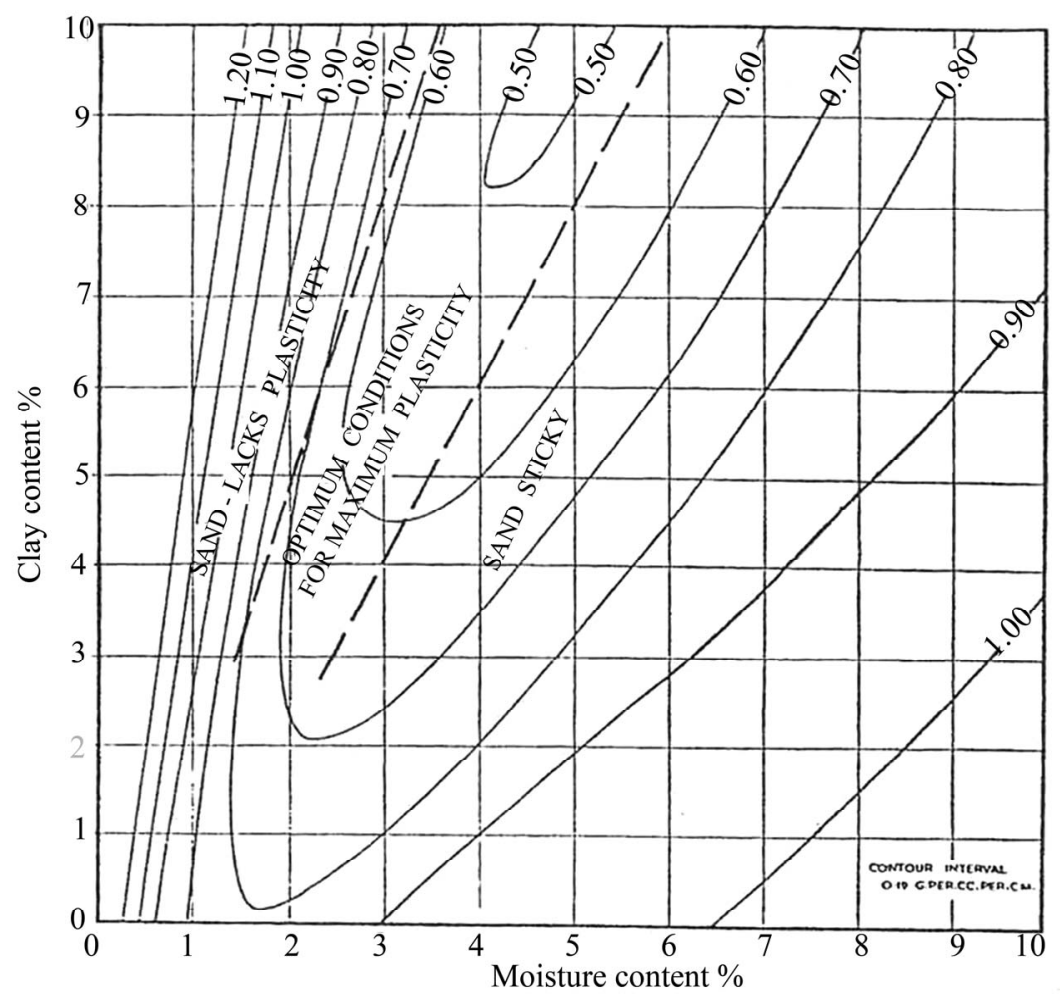

Figure 4. Showing the relation between the quality of the mould cavity and the bentonite (clay) to moisture content.

nates of this sand it is possible to decrease the bentonite /water ratio without changing the mould cavity properties and meanwhile increase the cooling rate and consequently change the DAS size and increase the mechanical properties. The holes in the carboceramics can absorb more moisture and as a consequence the solidification time will decrease. In this research, dual effects of using fins and carboceramics on the solidification time of the castings have been investigated.

\section{Experimental Procedure}

In the present work, the effect of moisture content on cooling rate of sand moulds made of carboceramics, and also the effect of fin on the solidification time have been investigated. To do this, after designing and constructing the pattern, the $\mathrm{K}$ thermocouple and Labview 2 software prepared.

\subsection{Moulding and Casting}

The pattern used in this research is seen in Figures $\mathbf{5}$ and 6. The mould cavity was prepared using $\mathrm{SiO}_{2}$ sand with various percentages of bentonite and moisture. Carboceramic sand was also used similar to $\mathrm{SiO}_{2}$ sand at different bentonite moisture ratios.

Also, Pep set and Dry Pep set methods were applied to prepare the mould. In this case, $1 / 2 \%$ resin and $1 / 2 \%$ catalyst were added to the sand and mixed for 5 minutes. In

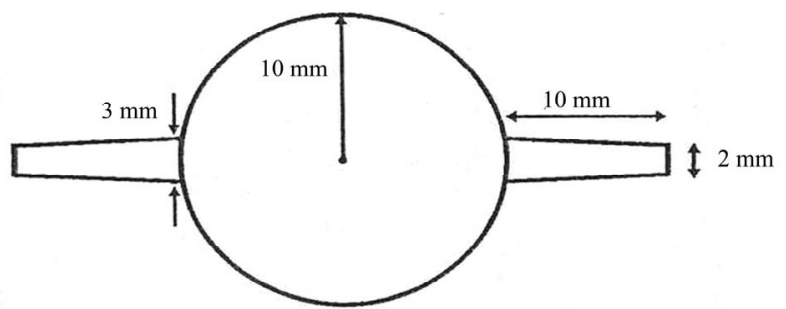

Figure 5. The cross section of the pattern.

Dry Pep set system, the mould prepared in the first step, was preheated in furnace in $800^{\circ} \mathrm{C}$ for two hours. A $25 \mathrm{~kg}$ medium induction furnace was used. The A356 and 6069 alloys were melted and all moulds were cast at $720^{\circ} \mathrm{C}$.

\subsection{Thermal Analysis}

All castings were thermally analyzed by Lab view II software. In this case, $\mathrm{K}$ type thermocouple was prepared as seen in Figure 7, and was installed with $25 \mathrm{~mm}$ distance from the casting bottom in the middle of the section. For any situation, at least two castings were produced and thermal analysis was carried out by computer.

The cooling curve and first derivative were drawn and analyzed. The solidification time of both castings were calculated from the curve of first derivative. The measurement was in a way that the time of beginning of the solidification in cooling curve is equal to the first jump in 

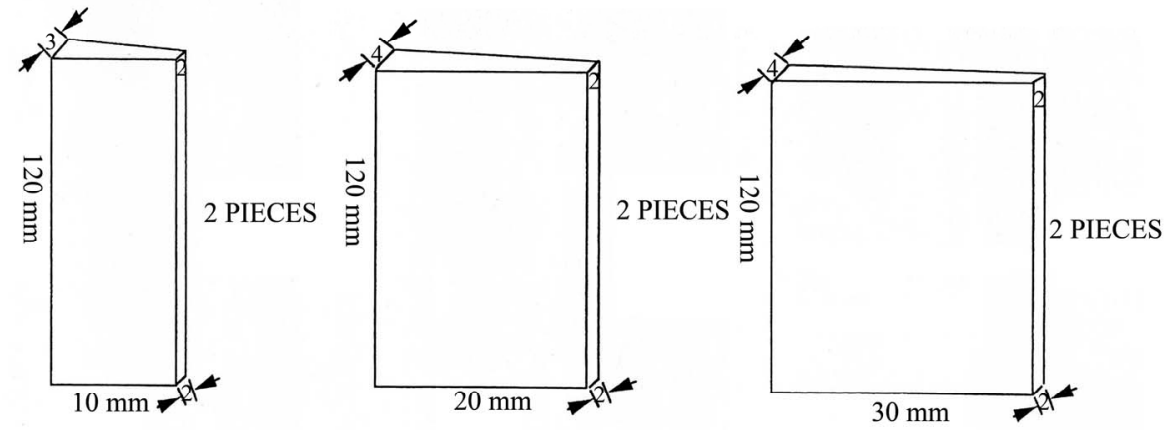

Figure 6. Dimensions of the three different used fins.

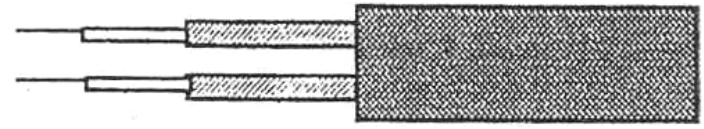

Figure 7. Schematic of the used K-type thermocouple.

the first derivative curve and the time of end of the solidification is where the base line and curve of eutectic section converge.

\subsection{Cooling Curves and their Derivatives}

Figure 8 indicates the curves of the samples of thermal analysis for alloy A356. As seen in the diagrams, the curves include two hypoeutectic and eutectic reactions. The cooling curves and first derivatives in alloys A356 and 6069 can be seen in Figure 9. The general behavior of alloy 6069 is like pure metal.

The solidification time of all moulds, cast at different conditions has been calculated from the first derivative.

\section{Results and Discussion}

\subsection{Mould Cavity}

The mould cavity is the main base in which the melt is cast. Because the molten metal flowing into the mould cavity has high temperature, many considerations should be taken into account. The most important cases include: permeability, mould hardness, wet and dry strength, mould resistance against thermal shocks, resistance against erosion of floating fluid, etc.

Physical and metallurgical properties of the moulds prepared in the project have been revised specifically. In the green sand moulds, by increasing the ratio of bentonite to moisture in the low strength area, low resistance against erosion will be forced. The only advantage of this part would be the faster heat transfer and consequently decreasing the local solidification time.

Because in this area, the main properties required by the mould namely resistance against erosion are so low, it is not recommended to work at this area and it is not possible to achieve the desirable properties through de- creasing the solidification time. Of course, as is seen this is evident in mould with $\mathrm{SiO}_{2}$ base. But if carboceramic sand is used, the added moisture not only moisturizes the sand surface, penetrates into the sand and therefore, thermal transference is accelerated. Hence the area for

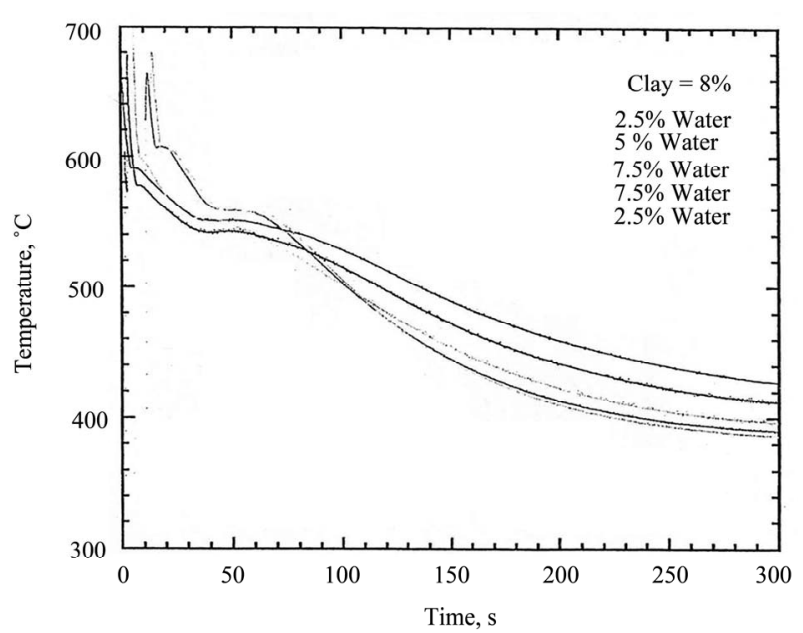

(a)

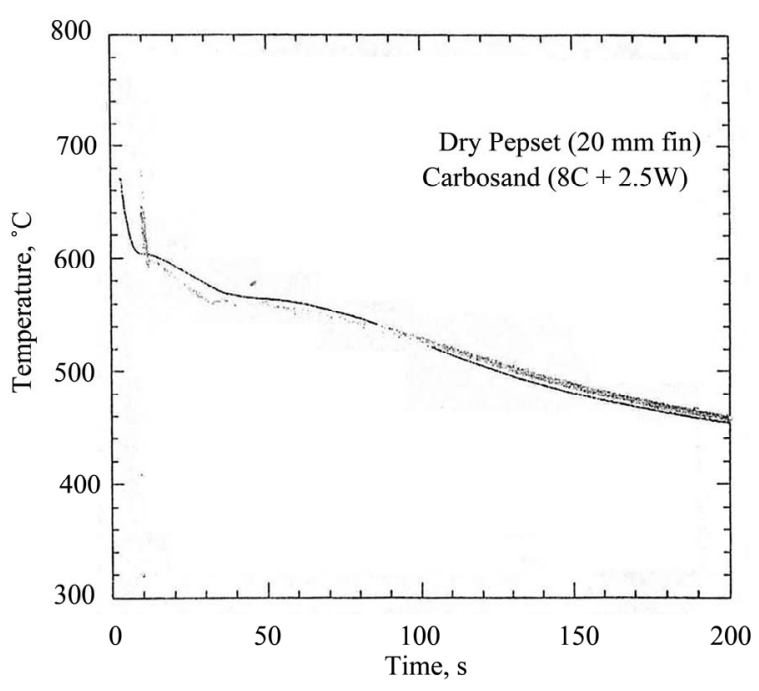

(b) 


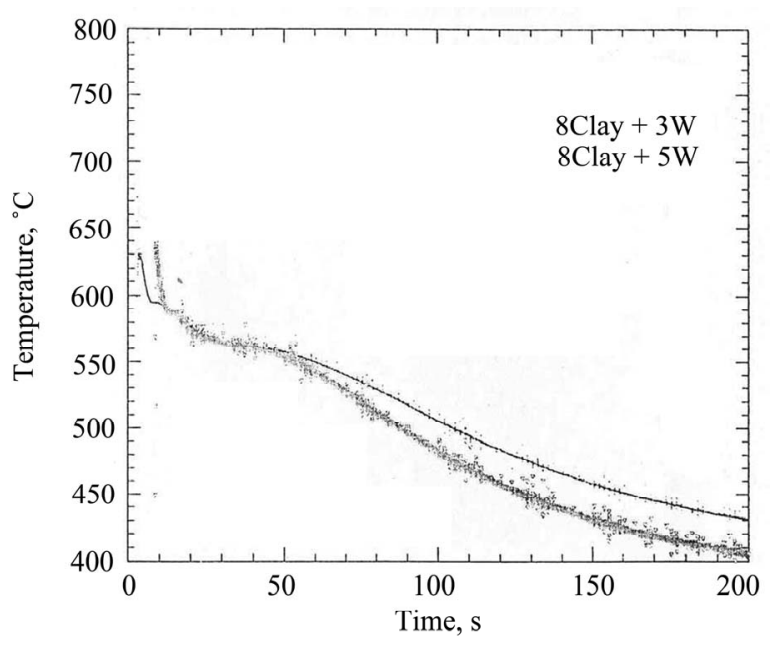

(c)

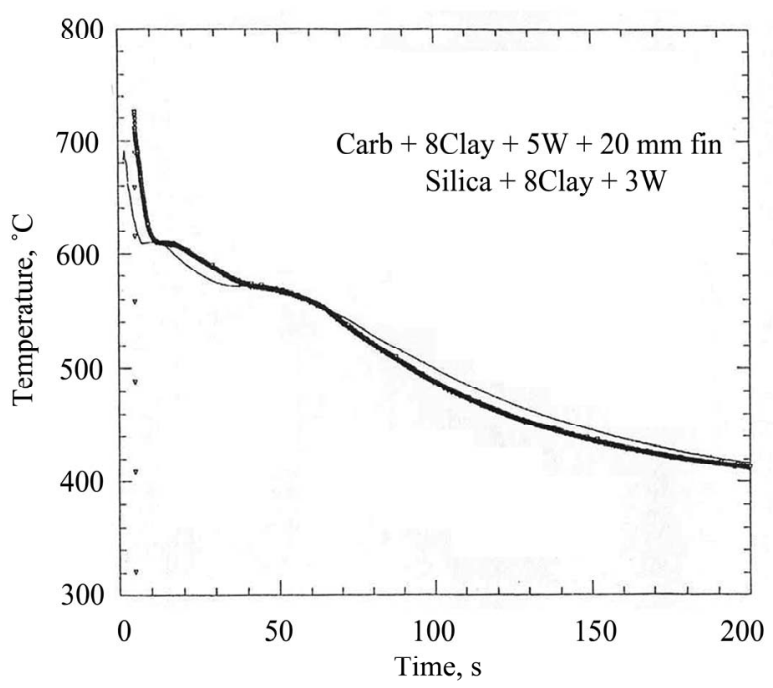

(d)

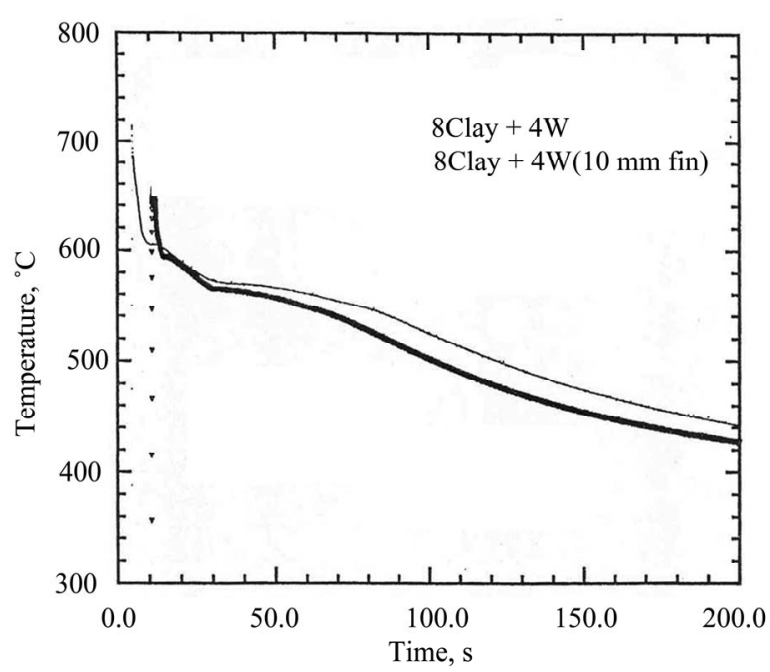

(e)

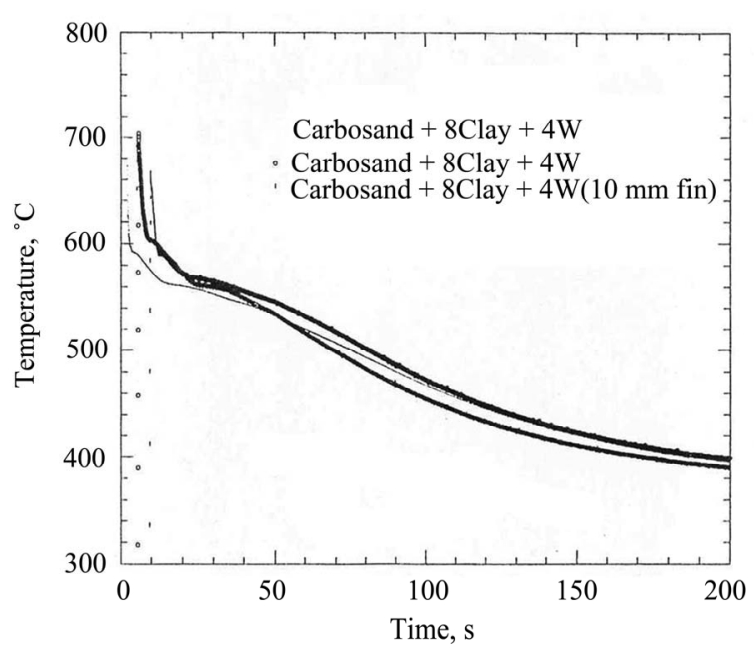

(f)

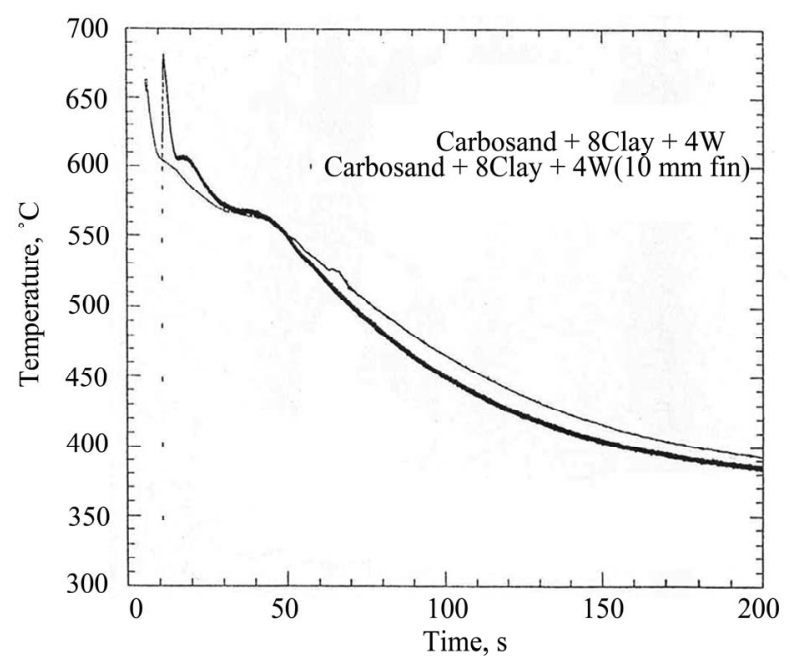

(g)

Figsure 8. Cooling curves of the castings with different water content for alloy A356.

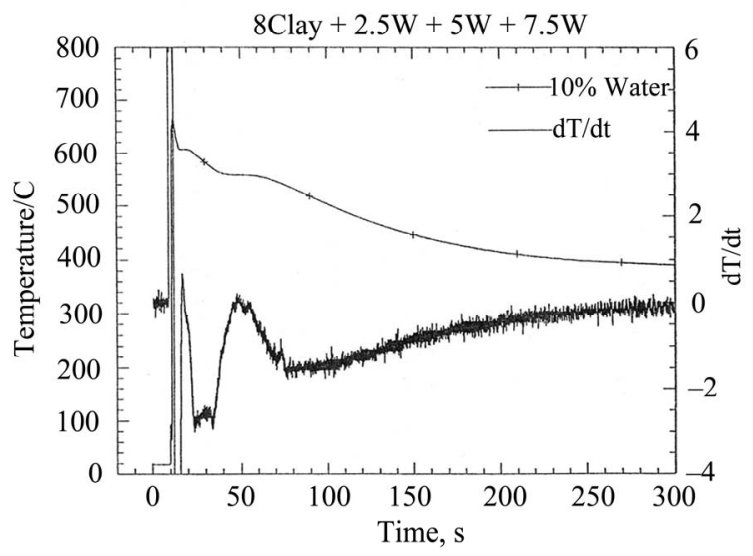

(a) 


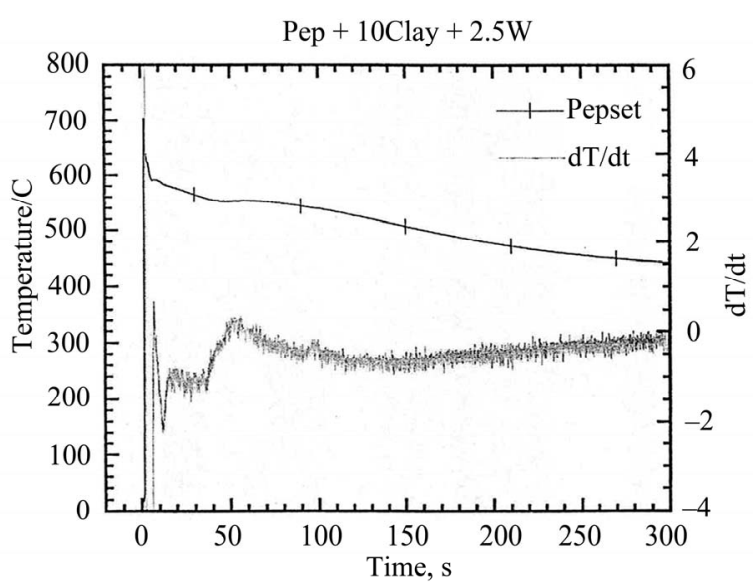

(b)

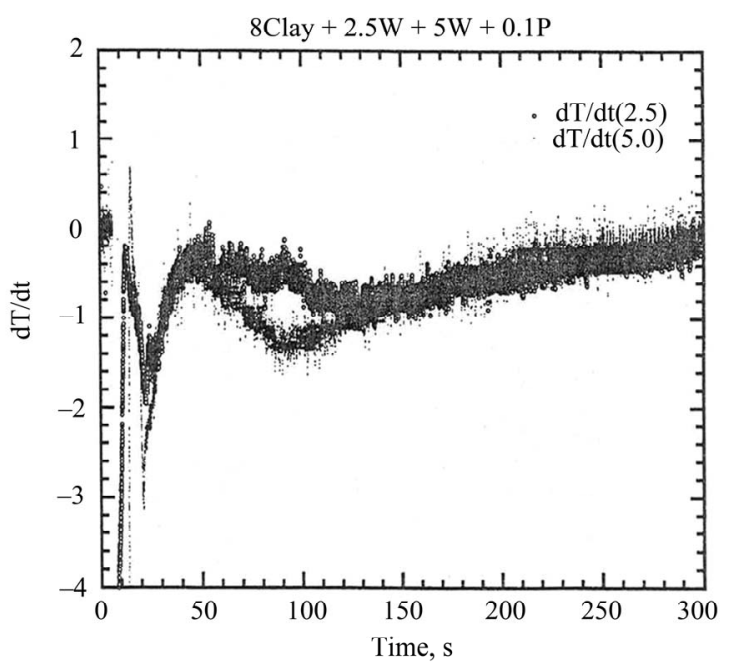

(c)

Figure 9. Cooling curves and first derivatives and the castings produced in different mould material and different constituents for alloys A356 and 6069.

using more moisture for carboceramics should be studied more, because in this mode, it is possible to decrease the local solidification time and to gain smaller DAS. It is clear that mechanical properties are increased following this mode.

In addition to above advantages, higher thermal conductivity of carboceramic containing $\mathrm{Al}_{2} \mathrm{O}_{3}$ is also of importance.

The unevenness of the primary surface produced by carboceramic sand, if its moisture is high, will be lower than that of moulds casted by $\mathrm{SiO}$ sand. To compensate this, one way is to change the granular distribution of carboceramic sand. Moulds with resin and catalysts are of greater strength and thermal conductivity is lower in these casts than the wet sand, and the solidification time of the castings is $25 \%$ higher than those moulded by wet sand.
When the moulds produced by chemical method are cooled in the furnace, the chemicals are removed and the solidification time is lengthened (Figure 10).

\subsection{Moulding Materials}

The speed of heat transfer from the mould cavity outwards is a function of type of moulding material and mould modulus. As seen, heat transfer is accelerated by increasing in moisture content and therefore the solidification time is decreased. This is due to water which is the factor for accelerating the heat transfer. Of course as is evident from Figure 4, moisture can be added only in a limited percentage. In some cases we may reach a point where the sand is stuck to the mould and will become sticky. Meanwhile, in this case, mould strength will decrease. Now if the mould thickness is low, we will enter a point where the sand elasticity, in other words, its formability will decrease. The reasonable metallurgical limit is when it is possible to maintain bentonite and water ratio around 2 . In this case we will have a rigid mould with high permeability (Figure 4).

In natural washed $\mathrm{SiO} 2$ sands, the particles of sand grains are not porous and the water added to the sand will wet the surface of the grains. And the adding bentonite to the humid sand is accompanied with activating the sodium ion of the bentonite. Consequently, the moulding materials with a low compaction exerted on them through hand, or machine, the desirable rigidity will be achieved.

On the other hand, in carboceramic sands, because of the porosity of the sand granules, the water penetrates into the porosity of the sand, and will change the heat transfer and cooling rate of the mould cavity and this is a very important fact (Figure 11).

As is clear in the diagram, carboceramic in addition to

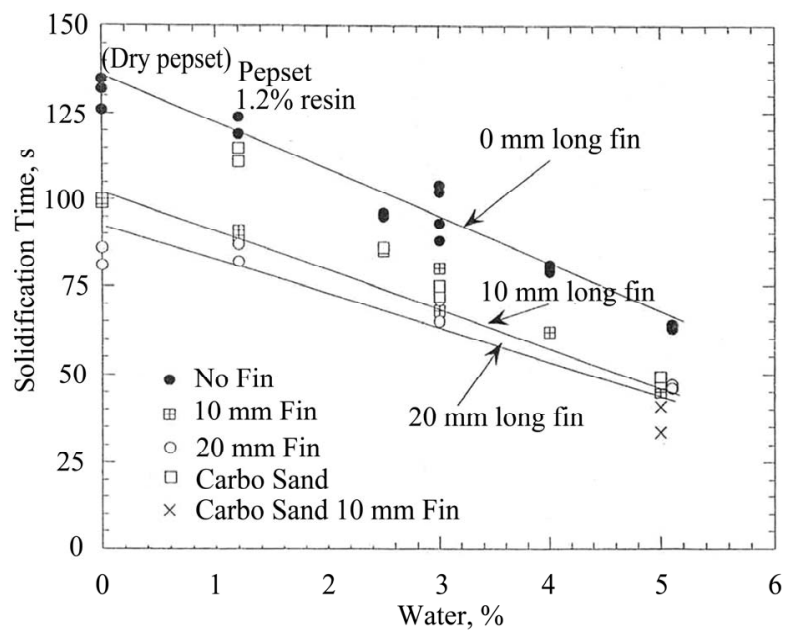

Figure 10. Variations of solidification time as a function of moisture content of the mould and the fin size. 

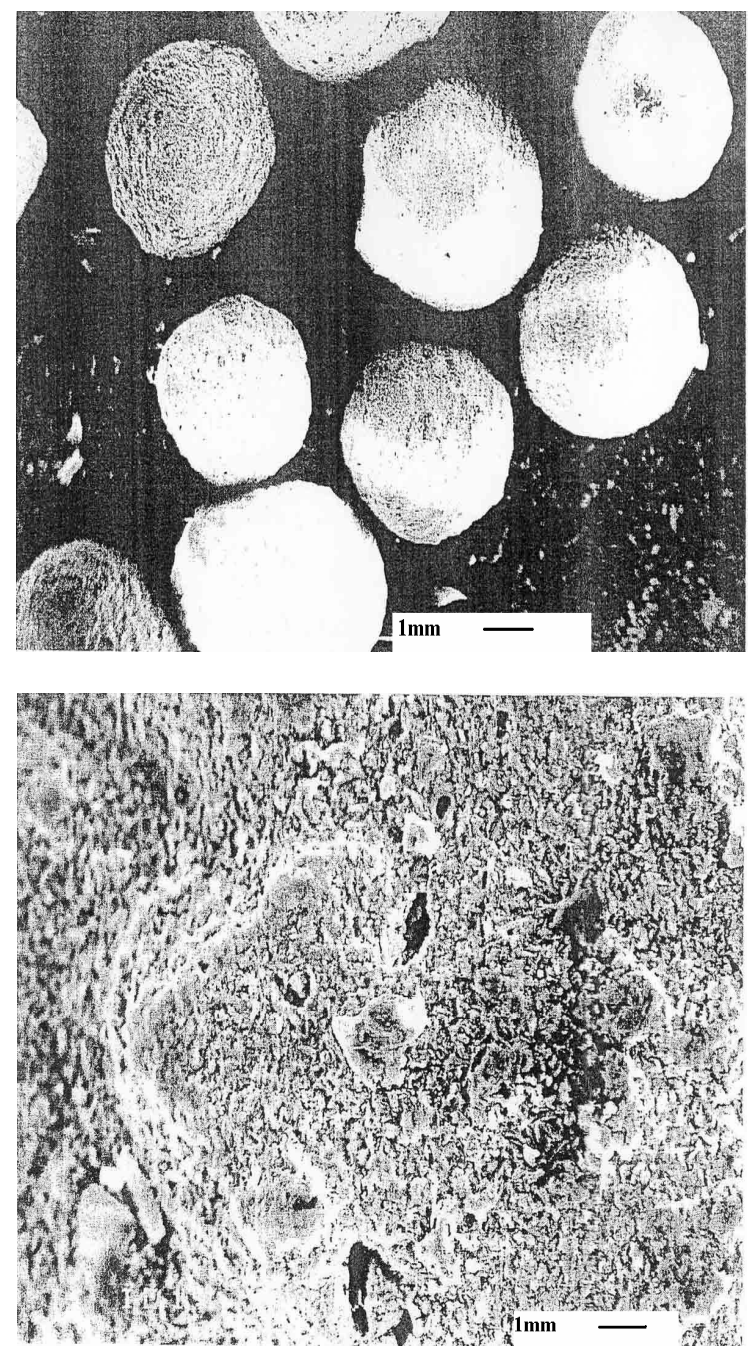

Figure 11. Morphology of the carboceramic sand which is indicated the high porous surface.

maintaining the mould's rigidity, absorbs moisture up to 5 percent. This is an advantage of this type of sand which has been less emphasized up to now.

The mould made of Pep Set indicates lower solidification time in comparison with wet sand implying the absence of moisture in that. This is evident also in dried Pepset mould, because in that, resin and catalyst bands are transformed and the sand is approximately dried (Figure 11).

\subsection{Fins}

Fin decreases the local solidification time. The main reason for decreasing the solidification time is the fact that the melt flowing into the mould, also fills the fins and considering the fact that the fin surface is where the heat transfers, and this is a broad and considerable surface, the casting solidified more quickly. This will de- crease the DAS and consequently increases the mechanical properties. What is important is that fins move isotherm temperature surfaces when solidifying and therefore lead to transfer, decrease, and desirable distribution or elimination of shrinkage porosity and this is so important in casting industry.

This research indicates that the solidification time will decrease up to $45 \%$ in case fins are used and this is good news for foundrymen.

\section{Conclusions}

1) Local solidification time is a function of moulding materials, casting thermal conductivity and fin dimensions.

2) Carboceramics will have good future in casting industry.

3) As the casting's thermal conductivity decreases, the fin effect increases.

4) The increase in the thermal conductivity of melt will increase fin effect.

5) The increase in superheating will increase the fin effect.

6) Fin's optimum thickness in practice is 0.1 half of the thickness.

7) The most appropriate length of fin is 4 times the half of casting thickness. In this situation, up to 70 percent of local solidification time has been recorded.

\section{REFERENCES}

[1] R. C. Creese and A. R. Sarfaraz, "Effect of Fins at Design of Junction in Castings," AFS Transactions, Vol. 2, 1987, pp. 1203-1215.

[2] V. S. Zolotorevsky, N. A. Belov and M. V. Glazoff, "Casting Aluminum Alloys," Elsevier Ltd., Amsterdam, 2007.

[3] W. C. Erickson, "Computer Simulation of Solidification," AFS Cast Metals, Vol. 8, 1980, pp. 89-97.

[4] R. M. Kotschi and C. R. Loper, "Effect of Chills and Cores on the Design of Junction in Simple Shape Castings," AFS Transactions, Vol. 4, 1976, pp. 301-304.

[5] D. Dispinar et al., "Degassing, Hydrogen and Porosity Phenomena in A356," Materials Science and Engineering, Vol. 527, No. 16-17, June 2010, pp. 3719-3725.

[6] H. Nguyen, "The Effects of Solidification Rates on Porosity Formation and Cast Microstructure of Aluminum Alloy A356," Grand Valley State University, Allendale, 2005.

[7] M. H. Kim and J. T. Berry, "Some Experimental Observation of the Effect of Fins on the Solidification of Pure Lead, Zinc, and Copper," Transactions of the American Foundrymen's Society, Vol. 97, May 1989, pp. 329-334.

[8] H. M. Guo et al., "Pressurized Solidification of SemiSolid Aluminum Die Casting Alloy A356," Journal of Al- 
loys and Compounds, Vol. 485, No. 1-2, 2009, pp. 812816. doi:10.1016/j.jallcom.2009.06.083

[9] R. C. Creese and A. R. Sarfaraz, "Effect of Fins on Casting Junction Design," AFS Transactions, Vol. 8, 1987, pp. 231-239.

[10] R. C. Creese and A. R. Sarfaraz, "Computer Wave Fronts Analysis of Fin Effect in L-shaped Castings," AFS Transactions, Vol. 8, 1988, pp. 61-68.

[11] R. C. Creese and A. R. Sarfaraz, "Fin Size Determination for Producing Sound Casting," AFS Transactions, Vol. 8, 1989, pp. 188-195.
[12] M. H. Kim, C. R. Loper and C. S. Kang, "Experimental Analysis of Wave Fronts in Standard Casting Sections and in Finned Castings," AFS Transactions, Vol. 2, 1985, pp. 418-424.

[13] Y. A. Gengel, "Introduction to Thermodynamics and Heat Transfer,” Mc-Grawhill, New York, 1997. 